PATRONES GeOMÉTRICOS, NUMÉRICOS Y VERBALES COMO INICIADORES DEL PROCESO DE GENERALIZACIÓN EN LA EDUCACIÓN BÁSICA PRIMARIA

\title{
Patrones geométricos, numéricos y verbales como iniciadores del proceso de generalización en la educación básica primaria ${ }^{1}$
}

\author{
Geometric patterns, numerical and verbal as initiators of the \\ process of generalization in basic primary education
}

\section{Os padrões geométricos, numérica e verbal como iniciadores do processo de generalização do ensino primário básico}

Recibido: mayo 2013

Aceptado: agosto 2013
Xiomara Corredor Santos ${ }^{2}$

Mónica Pineda Ballesteros ${ }^{3}$

Solange Roa Fuentes ${ }^{4}$

\section{Resumen}

En este escrito se analiza el desarrollo del proceso de generalización de estudiantes entre 9 y 12 años, a partir del estudio de situaciones sobre patrones en diferentes representaciones. Para esto se consideran las actividades básicas del proceso de generalización propuestas por Kaput en 1999: 1. Identificar similitudes en un conjunto de casos, 2. Extender el razonamiento propio más allá del rango en el cual se originó y 3 . Derivar resultados más amplios de casos particulares. Los resultados muestran que después de un proceso de instrucción los estudiantes logran identificar algunos patrones en secuencias numéricas y geométricas; sin embargo, tienen grandes dificultades para generalizarlos ya sea de manera verbal o mediante una expresión general.

Palabras clave: Aprendizaje; Procesos Cognitivos; Generalización; Patrones; Actividades básicas; Otras nociones de Educación Matemáticas; Sistemas de representación numérica y geométrica.

\begin{abstract}
In this paper we analyze the generalization process development of students between 9 and 12 years, from the study of situations on patterns in different representations. For this we consider the basic activities of the process of generalization proposed by Kaput in 1999: 1. Identify similarities in a set of cases, 2. Extending their reasoning beyond the range in which it originated and 3. Derive broader outcomes of particular cases. The results show that after a process of instruction, students are able to identify some patterns in numerical and geometric sequences, however, have great difficulty generalizing either verbally or by a general expression.
\end{abstract}

1 Artículo de Investigación.

2 Universidad Industrial de Santander. Colombia. Contacto: xiomy_1121@hotmail.com

3 Universidad Industrial de Santander. Colombia. Contacto: monypin23@gmail.com

4 Universidad Industrial de Santander. Colombia. Contacto: sroa@matematicas.uis.edu.co 
Keyword: Learning, Cognitive Processes, Generalization, Patterns, Core Activities, Other notions of Mathematics Education; Systems numeric and geometric.

\section{Resumo}

Neste artigo analisamos o desenvolvimento do processo de generalização dos alunos entre os 9 e os 12 anos, a partir do estudo de situações em padrões em diferentes representações. Para isso, considerar as atividades básicas do processo de generalização propostos por Kaput em 1999: 1. Identificar semelhanças em um conjunto de casos, 2 . Estendendo seu raciocínio para além da faixa na qual ela se originou e 3 . Obter resultados mais amplos de casos particulares. Os resultados mostram que, após um processo de instrução, os alunos são capazes de identificar alguns padrões em sequências numéricas e geométricas, no entanto, têm grande dificuldade em generalizar verbalmente ou por uma expressão geral.

Palabras clave: Aprendizagem, Processos Cognitivos, Generalização, Patterns, Núcleo de Atividades, outras noções de Educação Matemática; sistemas numéricos e geométricos.

\section{Introducción}

Tradicionalmente el estudio del álgebra se ha relacionado con la habilidad de los estudiantes al tratar con expresiones algebraicas y ecuaciones. Es común que diferentes miembros de la sociedad (padres de familia, estudiantes e incluso maestros) consideren que el estudio del álgebra se inicia en el tercer año de secundaria. Sin embargo como se propone en los estándares para matemáticas de la NCTM (National Council of Teachers of Mathematics, por sus siglas en inglés, 2000) el desarrollo del pensamiento algebraico debe iniciarse desde la etapa preescolar. Merino (2012) menciona que esto es necesario no sólo para facilitar su posterior estudio, sino además, para promover en los estudiantes un aprendizaje más comprensivo, profundo y complejo de las matemáticas escolares. Recientes investigaciones muestran que la propuesta de cambio curricular planteada por Early Algebra (Kaput, 2000; Blanton \& Kaput, 2005; Vasco, 2007), responde a estas necesidades, ya que no se trata de introducir un curso de álgebra en los primeros años escolares sino de promover una forma de pensar sobre las estructuras y relaciones entre objetos, que deben guiar el desarrollo del pensamiento matemático de los estudiantes desde edades tempranas (Vergel, 2010).
En este trabajo nos centramos en analizar y desarrollar el primer estándar propuesto para Álgebra por la NCTM este es: "Comprender patrones, relaciones y funciones" (NCTM, 2000, p.40). Para la etapa 3-5 el desarrollo de este estándar se caracteriza por el trabajo con variables y expresiones algebraicas a partir del estudio de patrones numéricos, geométricos, sus representaciones y las generalizaciones que se logran sobre ellos (NCTM, 2000). Entonces nuestro objetivo es hacer un primer acercamiento con grupos regulares de primaria, para estudiar el proceso de generalización que se realiza a partir de una secuencia (Radford, 2008); no sólo como un método para resolver situaciones específicas sino más bien, con la intensión de desarrollar las tres actividades básicas que propone Kaput (1999) como indispensables para el desarrollo del proceso de generalización.

Por tanto nos plantemos la siguiente pregunta:

¿Qué tipo de actividades realizan estudiantes entre 9 y 12 años al abordar el estudio de patrones numéricos, geométricos y sus representaciones? Y ¿Cómo dichas actividades potencian el proceso de generalización? 


\section{Elementos del marco conceptual}

A continuación describiremos elementos teóricos esenciales que fundamentan esta investigación. Cuando hablamos de "patrones" nos referimos a aquello que se repite con regularidad (Castro, 1995). La identificación de un patrón es una forma de generar una "generalización de un fenómeno"; respecto a esto Kaput (1999) menciona:

...extender deliberadamente el rango de razonamiento o comunicación más allá del caso o casos considerados, identificados explícitamente y exponiendo similitud entre casos, $\mathrm{o}$ aumentando el razonamiento o comunicación de un nivel donde el foco no son los casos o situaciones en sí mismos, sino los patrones, procedimientos, estructuras y las relaciones a lo largo y entre ellos. (p. 136)

Mediante un proceso de observación los estudiantes pueden identificar aquello que permanece constante y que puede ser generalizable. Kaput (1999) propone que este proceso requiere del desarrollo de tres actividades básicas: 1 . Identificar similitudes en un conjunto de casos, 2. Extender el razonamiento propio más allá del rango en el cual se originó y 3 . Derivar resultados más amplios de casos particulares. Estas actividades específicas fundamentan el diseño de las situaciones propuestas en nuestra investigación y de manera natural, se perciben en el trabajo de los estudiantes una vez ellos logran familiarizarse con los problemas de patrones en diferentes representaciones. El paso por cada actividad genera expresiones (gráficas o verbales) que les permiten predecir con precisión el comportamiento del patrón sin realizar una acción externa específica (realizar paso a paso cada caso).

El desarrollo de las Acciones específicas que guían nuestro método se basan en la recomendación propuesta en los estándares de la NCTM (2000) referente al estudio del álgebra como:

...una constante en el currículo desde la educación infantil en adelante, los docentes pueden ayudar a los estudiantes a construir una base sólida de aprendizaje y experiencia como preparación para un trabajo más sofisticado en el álgebra en los grados medio y superior. (p.37)

Por tanto es indispensable garantizar dentro del currículo experiencias que promuevan de manera regular y progresiva el desarrollo del proceso de generalización como una actividad propia del pensamiento matemático.

\section{Metodología}

El método que guía nuestro trabajo se desarrolla en tres fases, en ellas buscamos encontrar evidencias sobre las actividades básicas del proceso de generalización.

1. Prueba diagnóstica: Diseño, análisis y aplicación de una prueba diagnóstica a dos grupos de estudiantes de quinto primaria (un total 79 estudiantes).

2. Diseño y desarrollo de una secuencia de enseñanza: Teniendo en cuenta los resultados de la Fase anterior diseñamos y aplicamos durante dos horas semanales 5 actividades, con dos cursos de quinto primaria. Cada una de ellas cuenta con un análisis a priori que describe el tipo de acciones específicas que esperamos los estudiantes desarrollen, cómo dichas acciones se relacionan con las tres actividades básicas del proceso de generalización y el tipo de estrategias metodológicas que se tienen en cuenta en el desarrollo de cada actividad.

3. Entrevista Semi-Estructurada: Con el diseño y desarrollo de esta entrevista, buscamos determinar de manera más específica las actividades básicas del proceso de generalización que los estudiantes lograron alcanzar después de la fase anterior.

Con este método esperamos identificar las actividades básicas que requiere el proceso de generalización (Kaput, 1999), y a partir de ellas, plantear estrategias curriculares y didácticas específicas 
sobre cómo potenciar este proceso en los primeros años escolares.

\section{Algunos resultados}

En esta sección mostraremos algunos resultados obtenidos en la prueba diagnóstica que desarrollamos con un total de 79 estudiantes. Dicha prueba estaba conformada por tres situaciones, nos centraremos en la primera.

Situación 1. Los siguientes números forman una secuencia, encuentra los números faltantes.

\section{$3,5,9,15, \ldots, \ldots, 45$,}

Explica con tus propias palabras cómo encontraste los valores que faltaban.

Inventa dos secuencias diferentes, en donde no apa-

rezcan algunos de sus valores. Imagina que eres un profesor o profesora de matemáticas y prepara esta actividad para tus estudiantes. Explica cuál es la clave para encontrar los valores en cada secuencia y cómo la construiste.

Fuente: Elaboración propia

Al inicio del trabajo esperábamos que la mayoría de los estudiantes pudieran determinar sin dificultad el patrón que estaba generando los términos de la secuencia dada; sin embargo encontramos gran dificultad para que lograran entender en qué consistía el problema. La mayoría de los estudiantes no estaban familiarizados con este tipo de situaciones. Una vez se explicó en qué consistía el problema pudimos identificar las actividades propuestas por Kaput (1999):

1. Identificar similitudes. Los estudiantes intentan identificar qué se mantiene constante en los términos dados. Pero al no identificar el patrón que genera cada término crean patrones "sectorizados" que reducen el nivel de abstracción de la situación. Por ejemplo, toman los términos a partir de 15 y escriben la sucesión 15, 17, 19 [Agregado 17 y 19 en los espacios en blanco].

2. Extensión de razonamiento más allá del rango en que se origina. Al momento de expresar el patrón verbalmente, los estudiantes tienen dificultades para expresar aquello que ven en la secuencia numérica. Por ejemplo escriben "Sumándole a cada dígito de a dos veces hasta llegar al número final".

3. Derivar resultados amplios de casos particulares. Los estudiantes que logran desarrollar la actividad con éxito pueden encontrar otras secuencias de términos y expresar la manera general de generarlos.

Durante la presentación de este trabajo profundizaremos sobre el análisis de los resultados y sobre los aspectos que caracterizan las actividades básicas del proceso de generalización

\section{Referencias}

Castro, E. (1995). Exploración de patrones numéricos mediante configuraciones puntuales. (Tesis Doctoral). Granada: Comares.

Kaput, J. (1999). Teaching and learning a new algebra. En E. Fennema y T. Romberg (Eds.), Mathematics classroom that promote understanding (pp. 133-155). Mahwah, NJ: Erlbaum.

Merino, E. (2012). Patrones y representaciones de alumnos de $5^{\circ}$ de educación primaria en una tarea de generalización (Tesis de Maestría). Universidad de Granada, Granada, España.

National Council of Teacher of Mathematics. (NCTM). (2000). Principles and standards for school mathematics. Reston, VA: Autor.

Vergel, C. (Octubre, 2010). La perspectiva de cambio curricular Early-Algebra como posibilidad para desarrollar el pensamiento algebraico en escolares de educación primaria: Una mirada al proceso matemático de generalización. G. Obando (Presidencia), $11^{\circ}$ Encuentro Colombiano Matemática Educativa, Bogotá, Colombia. 